\title{
Impact of laterally non-uniform carrier lifetime on photoconductance-based lifetime measurements with self-consistent calibration
}

\author{
W. S. Liang*, Klaus J. Weber and Y. L. Ren \\ Centre for Sustainable Energy Systems, College of Engineering and Computer Science, The Australian National University, Canberra, \\ Australian Capital Territory 0200, Australia
}

\begin{abstract}
The impact of laterally non-uniform carrier lifetime on the determination of the lifetime from photoconductance-based measurements, based on the self-consistent method proposed by Trupke and Bardos, is investigated using a simple model. It is shown that the method can result in an overestimation of the mean lifetime, with the magnitude of the error mainly dependent on the distribution of the effective lifetime across the area sensed by the photoconductance coil. Although in many cases the error introduced will be relatively small (in the order of 5\% or less), much larger errors can result in some cases, such as for samples that feature small areas with a significantly higher than average lifetime. The error can be eliminated through independent measurement of the sample optical properties. Experimental measurements confirm the model predictions. Copyright (C) 2012 John Wiley \& Sons, Ltd.
\end{abstract}

\section{KEYWORDS}

lifetime measurement; photoconductance; self-consistent; non-uniform lifetime

* Correspondence

Wensheng Liang, Centre for Sustainable Energy Systems, College of Engineering and Computer Science, The Australian National University, Canberra, Australian Capital Territory 0200, Australia.

E-mail: wensheng.liang@anu.edu.au

Received 20 November 2011; Revised 21 March 2012; Accepted 4 May 2012

The use of photoconductance (PC) measurements to determine the effective carrier lifetime $\tau_{\text {eff }}$ in silicon samples is a wellestablished experimental technique [1-8]. This technique is also used for converting a measured photoluminescence (PL) signal into a spatially averaged absolute excess carrier concentration in PL measurements (quasi-steady-state PL $[9,10]$ and Suns-PL [11]) and calibrating PL images to obtain quantitative carrier lifetime images [12]. The PC technique measures changes in the conductivity of the sensed area of a sample due to the generation of excess carriers upon illumination from a light source. Using the appropriate mobility model, the excess carrier concentration $\Delta n$ in the wafer is extracted from the signal of the sensed area [1]. In a typical experimental setup, the sensed area is a circular area, and the analysis assumes that the carrier lifetime is uniform over this area.

There are three basic approaches to measuring the lifetime using PC measurements. For a sample with specific lifetime $\left(\tau_{\text {eff }}\right)$, lifetime measurement approach can be selected by changing the flash turn-off time $\left(\tau_{\text {flash }}\right)$. In the transient model (PC decay), it is assumed that light generation is zero and that excess carriers decay towards their equilibrium value. This model therefore requires the use of a light source that can be cut off much more quickly (in other words, with a shorter decay time) than the carrier lifetime of the sample. In the quasi-steady-state PC (QSSPC) model, light generation is assumed to be constant, which requires that the rate of decay of the light intensity is much slower than the carrier lifetime of the sample $\left(\tau_{\text {eff }} / \tau_{\text {flash }}<0.1\right)$ [13]. The generalised model proposed by $\mathrm{H}$. Nagel is more powerful, allowing the determination of carrier lifetime even if neither of the above two conditions is satisfied [13]. It is most commonly used for PC measurements as it will in principle always result in the correct effective lifetime, provided that all required input parameters are accurately known.

The generalised relation between $\tau_{\text {eff }}$ and $\Delta n$ is given by

$$
\tau_{\text {eff }}=\frac{\Delta n(t)}{G(t)-\frac{\mathrm{d} \Delta n(t)}{\mathrm{d} t}}
$$

where $G(t)$ is the time-dependent generation rate of excess carriers per unit volume within the sample. 
Obtaining correct lifetimes requires knowledge of the optical properties of the sample, because $G(t)$ must be known. $G(t)$ can be determined experimentally from the following relation:

$$
G(t)=\frac{\phi_{\gamma}}{d} \cdot(1-T)\left(1-R_{\mathrm{f}}\right)
$$

where $\phi_{\gamma}$ is the incident photon flux, $R_{\mathrm{f}}$ is the front surface reflectance of the sample, $T$ is the transmittance of sample, which is very small for most real test samples, and $d$ is the sample thickness. Equation 2 can also be written as follows:

$$
G(t)=f_{\mathrm{s}} \cdot G_{\mathrm{rel}}(t)
$$

where $G_{\mathrm{rel}}(t)=\phi_{\gamma} / d$ is the relative generation rate, which can be determined from measurements of the photon flux and the sample thickness, and $f_{\mathrm{s}}=(1-T)\left(1-R_{\mathrm{f}}\right)$ is a scaling factor. Determination of the correct $G(t)$ therefore requires knowledge of $R_{\mathrm{f}}$ and $T$. Commonly, $f_{\mathrm{s}}$ can be extracted by comparing transient measurements with QSSPC measurements.

Trupke and Bardos [14] have described a self-consistent calibration method for PC and PL measurements, which obviates the need for an independent determination of sample reflectance and transmittance. The method is based on the fact that if a suitable symmetric light pulse is applied but the incorrect scaling factor is used, a hysteresis loop will appear in the $\tau_{\text {eff }}(\Delta n)$ versus $\Delta n$ curve. Elimination of the hysteresis occurs when the correct scaling factor is applied. However, this calibration method assumes a uniform carrier lifetime of the sample over the sensed area, a condition that is frequently violated in practice. This paper investigates how the accuracy of this method will be affected if the condition of lateral uniform lifetime is not met.

We investigated the impact of laterally non-uniform carrier lifetime on the calculated lifetime injection level curves using a simple numerical method, as was carried out by Trupke and Bardos [14]. For simplicity, we assume that the sensed area consists of two distinct regions with different lifetimes and relative areas and that the carrier lifetime in each region is independent of injection level. The sample thickness is $200 \mu \mathrm{m}$. A Gaussian light pulse is used for sample illumination, with a peak intensity $\phi_{\gamma \max }=2 \times 10^{17} \mathrm{~cm}^{-2} \mathrm{~s}^{-1}$ and a full width at half maximum of $5 \mathrm{~ms}$.

It should be noted that although the results presented below were obtained with a Gaussian light pulse, simulations were also carried out using a triangular light pulse to estimate the impact of the light pulse shape. The results from two illumination pulses were similar. Shapes of Gaussian and triangular pulses are shown in Figure 1.

In the simulations, the 'correct' scaling factor is set to $f_{\mathrm{s}}=1$; a value greater than 1 means that the carrier generation in the sample is overestimated, whereas a value less than 1 means that it is underestimated.

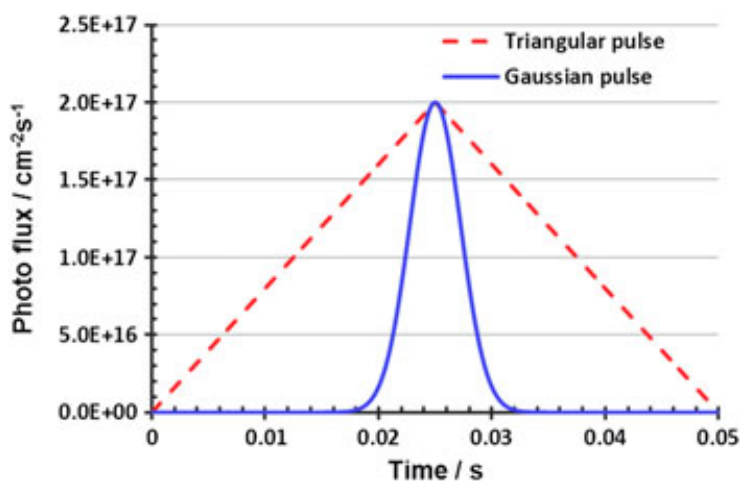

Figure 1. Gaussian and triangular illumination pulses used for simulations. Both pulses have the same peak intensity $\phi_{\gamma \max }=2 \times 10^{17} \mathrm{~cm}^{-2} \mathrm{~s}^{-1}$ and pulse width of $0.05 \mathrm{~s}$.

Figure 2(a) shows the simulated results for the case where the lifetimes of the two regions are the same $(300 \mu \mathrm{s})$ with scaling factors $0.9,1.0$ and 1.1 , respectively. In this case, $f_{\mathrm{s}}=1$ gives the correct lifetime and eliminates hysteresis, as expected.

Figure 2(b) illustrates the impact of non-uniform lifetime. In this case, $90 \%$ of the sensed area has a high lifetime of $300 \mu \mathrm{s}$, and $10 \%$ has a low lifetime of $30 \mu \mathrm{s}$. This situation is often encountered in practice, for example, as a result of the local diffusion of a contaminant during high temperature processing. In this case, hysteresis is minimised by choosing $f_{\mathrm{s}}=0.92$, which means that carrier generation in the sample is underestimated by $8 \%$. The effective lifetime extracted from the measurement is $297 \mu \mathrm{s}$, which is larger than the area-weighted (mean) effective lifetime of the sample $(273 \mu \mathrm{s})$ by $9 \%$. This error in the effective lifetime in turn would cause the same relative error in calibrated lifetime images obtained from PL measurements.

For the simulation scenario stated earlier, although $f_{\mathrm{s}}=0.92$ is the calibrated scaling factor according to the self-consistent calibration method, the correct scaling factor remains $f_{\mathrm{s}}=1$, because it is independent of lifetime distribution above the sensed area. The simulation results are shown in Figure 2(b). For $f_{\mathrm{s}}=1$, the mean lifetime value, obtained by taking the average from the two halves of the curve at each injection level, is very similar to the area-weighted lifetime over the whole $\Delta n$ range. In this case, we can argue that if we are aware of the optical parameters of sample surface, the correct lifetime curve is able to be obtained by the generalised model.

We investigated several other scenarios in order to assess the impact on the calculated effective lifetime. In every case, it was possible to almost completely eliminate hysteresis from the resulting $\tau_{\text {eff }}(\Delta n)$ versus $\Delta n$ curves through the choice of a suitable value of $f_{\mathrm{s}}$. Three illustrative scenarios (including that shown in Figure 2(b)) are summarised in Table I. The second scenario $(50 \%$ of the area with a $300-\mu$ s lifetime and $50 \%$ of the area with a $150-\mu$ s lifetime) corresponds to the case of a gradual but significant change in the lifetime across the sample, which 

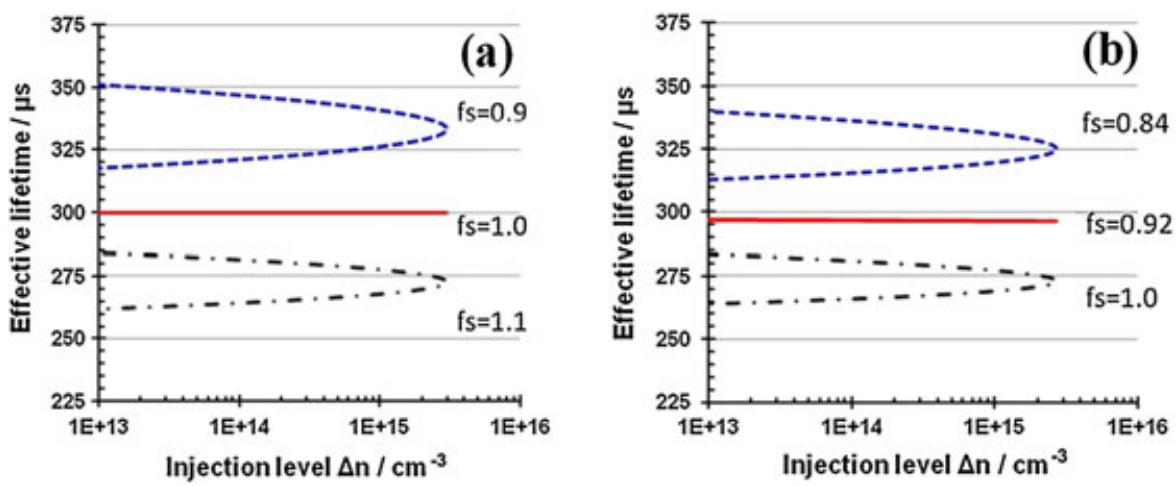

Figure 2. Calculated curves of $\tau_{\text {eff }}(\Delta n)$ versus $\Delta n$ for different values of the scaling factor $f_{\mathrm{s}}$ for (a) a sample with a uniform lifetime of $300 \mu \mathrm{s}$ and (b) a sample with two regions with different lifetimes (region I: $\tau=300 \mu \mathrm{s}, 90 \%$ of total area; region II: $\tau=30 \mu \mathrm{s}, 10 \%$ of total area).

Table I. Scaling factor $f_{\mathrm{s}}$ that minimises hysteresis of $\tau_{\text {eff }}(\Delta n)$ versus $\Delta n$ curves and resulting ratio of calculated mean lifetime to actual mean lifetime for three illustrative scenarios with different lifetime distributions.

\begin{tabular}{|c|c|c|c|c|c|}
\hline \multirow{2}{*}{$\begin{array}{l}\text { Region I } \\
{[\tau(\mu \mathrm{s}) / \text { area }(\%)]}\end{array}$} & \multirow{2}{*}{$\begin{array}{c}\text { Region II }[\tau \\
(\mu \mathrm{s}) / \text { area }(\%)]\end{array}$} & \multicolumn{2}{|c|}{ Gaussian light pulse } & \multicolumn{2}{|c|}{ Triangular light pulse } \\
\hline & & $f_{\mathrm{s}}$ & $\tau_{\text {cald }} / \tau_{\text {mean }}$ & $f_{\mathrm{s}}$ & $\tau_{\text {cald }} / \tau_{\text {mean }}$ \\
\hline $300 / 90$ & $30 / 10$ & 0.92 & 1.09 & 0.92 & 1.09 \\
\hline $300 / 50$ & $150 / 50$ & 0.91 & 1.11 & 0.90 & 1.11 \\
\hline $300 / 10$ & $30 / 90$ & 0.33 & 3.07 & 0.34 & 2.95 \\
\hline
\end{tabular}

may have multiple causes in practice, such as non-uniform quality of the starting material or contamination arising from high temperature treatments. Again, minimising hysteresis results in a modest but significant overestimate of the area-weighted lifetime. The third scenario (a small area with a high lifetime and a large area with a much lower lifetime) is the least likely to be encountered in practice, but it has the most dramatic effect of the estimated lifetime. In this case, the effective lifetime is overestimated by more than a factor of 3 . Such a situation could be encountered, for example, in multicrystalline silicon, where a small, well-passivated grain may be surrounded by several 'bad' grains.

We conclude that the determination of the effective sample lifetime using the technique of Trupke and Bardos [14] can lead to an overestimation of the area-weighted lifetime, if the lifetime over the sensed area is not uniform. This is because regions with a higher lifetime will effectively be weighted highly disproportionately in the calculation of the effective lifetime, as a result of the fact that the excess carriers in these regions decay more slowly than in the low lifetime regions. As is evidenced by the results in Table I, the magnitude of this effect depends on how the effective lifetime is distributed across the sample and is largest in areas that consist mostly of low lifetime regions, with some small regions of much higher lifetime.

The shape and duration of the light pulse also have some impact on the effective lifetime curves. For sufficiently short light pulses, elimination of hysteresis through the choice of the appropriate value of $f_{\mathrm{s}}$ is sometimes no longer possible. However, the above conclusions hold regardless of the shape of the light pulse.

In order to verify the predicted effects, we conducted PL imaging measurements using a LIS-R1 system from BT Imaging Pty Ltd located in NSW Australia, on a wafer of initially quite uniform and high lifetime. All measurements were conducted with an illumination wavelength of $808 \mathrm{~nm}$. A p-type shiny-etched Float Zone wafer with a crystal orientation of (100), a $0.8-\Omega \mathrm{cm}$ resistivity and a $300-\mu \mathrm{m}$ thickness was passivated by $50 \mathrm{~nm} \mathrm{SiO}_{2}$ grown in $1000^{\circ} \mathrm{C}$ followed by 30-min nitrogen in situ anneal and 30 -min forming gas anneal at $400^{\circ} \mathrm{C}$. $\mathrm{HF}$ fuming on half of the rear side is used to produce a low lifetime region. Effective lifetimes in two regions were 460 and $23 \mu$ s at injection level of $\Delta n=1 \times 10^{15} \mathrm{~cm}^{-3}$ with reflectance of $19 \%$. Fuming the rear of the sample has no effect on $G(t)$. First, lifetime images and curves under different illumination intensity for both the left, high lifetime area and right, low lifetime area were measured. For each measurement, lifetime calibration was carried out using the WCT120 PC system built into the PL equipment, and the sample reflectance was adjusted until hysteresis in the resulting $\tau_{\text {eff }}(\Delta n)$ versus $\Delta n$ curve was minimised, which occurred for $R_{\mathrm{f}}=19 \%$. The symmetric light pulse used for PL lifetime calibration was an exponentially rising and falling waveform with a frequency of $3.3 \mathrm{~Hz}$ and a peak amplitude corresponding 
to an incident photon flux of $2 \times 10^{17} \mathrm{~cm}^{-2} \mathrm{~s}^{-1}$. With this (correct) calibration, the average lifetime in a selected area from the high lifetime half of the sample, indicated by the dashed blue square in Figure 3, was measured at several photon fluxes using PL.

Secondly, the sample was positioned so that $50 \%$ of the sensing coil was covered by the high lifetime area and $50 \%$ by the low lifetime area, as shown by the red dashed circle in Figure 3. Lifetime calibration was again carried out, and hysteresis was minimised in the resulting $\tau_{\text {eff }}(\Delta n)$ versus $\Delta n$ curve. This time, minimising hysteresis required a (unrealistic) value of $R_{\mathrm{f}}=52 \%$. Again, the average lifetime in the same selected area from the 'good' half of the sample was measured at the same photon flux values.

It is known that measurements of the effective lifetime can be inaccurate if fast light transients are used and the sample surfaces display a high recombination velocity [15], and this could conceivably result in some errors in the measured effective lifetime of the low lifetime region. However, in our measurements and simulations, the exact value of the effective lifetime of this region has little influence on the results obtained because it is substantially lower than that of the high lifetime region.

In Figure 4, red squares represent the 'correct' measured effective lifetime. The lifetime of the same area obtained when the sensing coil straddled both regions is indicated by blue triangles. In this case, the measured lifetime, obtained using the self-consistent method with $R_{\mathrm{f}}=52 \%$, displays a $\sim 50 \%$ overestimation at any injection level. In practice, it would of course be obvious that a value of $R_{\mathrm{f}}=52 \%$ is unrealistic. The green crosses show the corresponding results from a simulation of this measurement, based on the measured $\tau_{\text {eff }}$ $(\Delta n)$ versus $\Delta n$ dependence of the two regions. The good fit between green crosses and blue triangles provides strong support for the model.

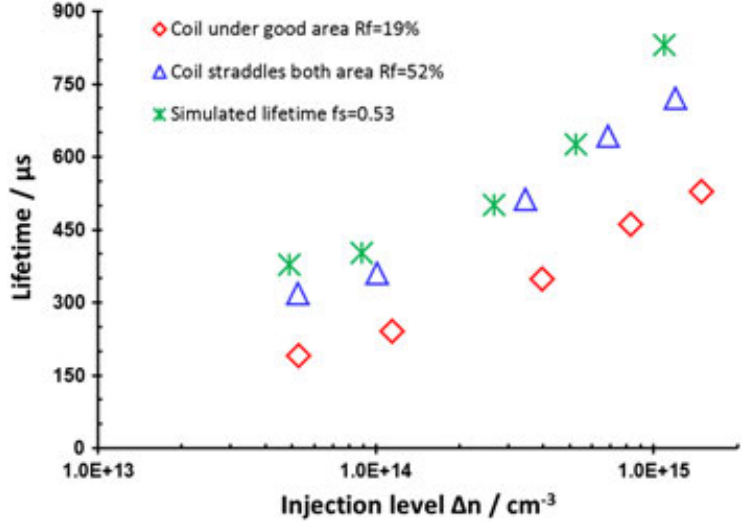

Figure 4. Experimentally determined and modelled lifetime of a high lifetime region of the sample investigated (blue dashed square in Figure 3 ) at various injection levels. Red squares: experimentally determined (correct) lifetime $\left(R_{\mathrm{f}}=\right.$ 19\%). Blue triangles: experimentally determined lifetime obtained by using the self-consistent calibration with the sensing coil straddling the high and low lifetime regions $\left(R_{\mathrm{f}}=52 \%\right)$. Green crosses: lifetime predicted from the model with the coil straddling the high and low lifetime regions $\left(R_{\mathrm{f}}=47 \%\right)$.

In conclusion, for the self-consistent determination of the effective lifetime based on the method of Trupke and Bardos [14], it is important that the sample lifetime is known to be laterally uniform. If this is not the case, application of the method could result in a significant overestimation of the lifetime. In such cases, the use of measured or reliable values of the optical parameters is likely to result in a more accurate determination of the mean lifetime.

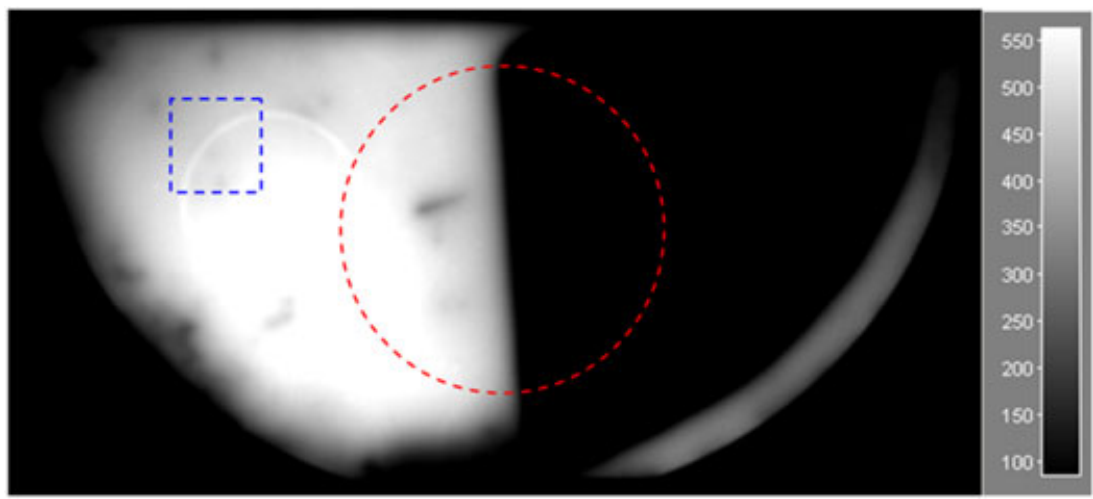

Figure 3. Effective lifetime image of a half-wafer produced using a calibrated photoluminescence measurement. The rear side of the right half part was HF fumed to obtain a low lifetime area. Bright edges on the right half are the result of incomplete removal of $\mathrm{SiO}_{2}$ in this region. The lifetimes of the left and right regions were 460 and $23 \mu$, respectively, at an injection level of $\Delta n=1 \times 10^{15} \mathrm{~cm}^{-3}$, with a reflectance of $19 \%$. The blue dashed square shows the region for which the average lifetime was calculated (see Figure 4), and the red dashed circle indicates the extent of the region sensed by the photoconductance coil. 


\section{ACKNOWLEDGEMENTS}

The authors gratefully acknowledge the helpful discussions with Dr Daniel Macdonald and the help with the lifetime measurements by Mr Nicholas Grant. The PhD scholarship for W. Liang from the China Scholarship Council and the Australian National University is gratefully acknowledged.

\section{REFERENCES}

1. Sinton RA, Cuevas A. Contactless determination of current-voltage characteristics and minority carrier lifetimes in semiconductors from quasi-steady-state photoconductance data. Applied Physics Letters 1996; 69(17): 2510.

2. Cuevas A, Stocks M, Macdonald D, Sinton R. Applications of the quasi-steady-state photoconductance technique. The 2nd World Photovoltaic Solar Energy Conference and Exhibition, Vienna, 1998.

3. Sinton RA, Cuevas A, Stuckings M. Quasi-steady-state photoconductance, a new method for solar cell material and device characterization. The 25th IEEE Photovoltaic Specialist Conference. Washington, DC, 1996.

4. Cuevas A, Macdonald D. Measuring and interpreting the lifetime of silicon wafers. Solar Energy 2004; 76: 255 .

5. Swirhun JS, Sinton RA, Forsyth MK, Mankad T. Contactless measurement of minority carrier lifetime in silicon ingots and bricks. Progress in Photovoltaics: Research and Applications 2011; 19:313.

6. Cuevas A, Sinton RA. Prediction of the open-circuit voltage of solar cells from the steady-state photoconductance. Progress in Photovoltaics: Research and Applications 1997; 5:79.
7. Cuevas A, Recart F. Capacitive effects in quasisteady-state voltage and lifetime measurements of silicon devices. Journal of Applied Physics 2005; 98:074507.

8. Kerr MJ, Cuevas A. Generalized analysis of quasi-steady-state and transient decay open circuit voltage measurements. Journal of Applied Physics 2002; 91: 399.

9. Trupke T, Bardos RA. Photoluminescence: a surprisingly sensitive lifetime technique. The 31st IEEE Photovoltaic Specialist Conference, Orlando, USA, 2005.

10. Trupke T, Bardos RA, Hudert F, Würfel P, Zhao J, Wang A, Green MA. Effective excess carrier lifetimes exceeding 100 milliseconds in float zone silicon determined from photoluminescence. The 19th European Photovoltaic Solar Energy Conference and Exhibition. Paris, France, 2004.

11. Trupke T, Bardos RA, Abbott MD, Cotter JE. Suns-photoluminescence: contactless determination of current-voltage characteristics of silicon wafers. Applied Physics Letters 2005; 87, 093503.

12. Herlufsen S, Schmidt J, Hinken D, Bothe K, Brendel R. Photoconductance-calibrated photoluminescence lifetime imaging of crystalline silicon. Physica Status Solidi-Rapid Research Letters 2008; 2(6), 245.

13. Nagel H, Berge C, Aberle AG. Generalized analysis of quasi-steady-state and quasi-transient measurements of carrier lifetimes in semiconductors. Journal of Applied Physics 1999; 86(11), 6218.

14. Trupke T, Bardos RA. Self-consistent determination of the generation rate from photoconductance measurements. Applied Physics Letters 2004; 85(16): 3611.

15. Sinton RA, Trupke T. Limitations on dynamic excess carrier lifetime calibration methods. Progress in Photovoltaics: Research and Applications 2012; 20:246. 